15 | 1998

Les dimensions sociales et économiques du développement local et la décentralisation en Afrique au Sud du Sahara

\title{
Chefs et Projets au Village (Niger)
}

Jean-Pierre Olivier de Sardan

\section{(2) OpenEdition}

\section{Journals}

Édition électronique

URL : http://journals.openedition.org/apad/563

DOI : 10.4000/apad.563

ISSN : 1950-6929

Éditeur

LIT Verlag

Édition imprimée

Date de publication : 1 mai 1998

Référence électronique

Jean-Pierre Olivier de Sardan, «Chefs et Projets au Village (Niger) », Bulletin de l'APAD [En ligne], 15 |

1998, mis en ligne le 20 décembre 2006, consulté le 08 septembre 2020. URL : http://

journals.openedition.org/apad/563; DOI : https://doi.org/10.4000/apad.563

Ce document a été généré automatiquement le 8 septembre 2020.

Bulletin de l'APAD 


\title{
Chefs et Projets au Village (Niger)
}

\author{
Jean-Pierre Olivier de Sardan
}

1 Ce texte a également été publié dans la série des Working Papers on African Societies $\left(\mathrm{n}^{\circ} 28\right.$, 1998, Berlin : Das Arabische Buch).

2 Un système politique local assez particulier caractérise le Niger. Il fait la part belle aux "chefferies", situées au centre des enjeux de pouvoir et d'influence en milieu rural mais qui "règnent" avec une efficacité somme toute limitée sur des sociétés villageoises peu organisées et difficilement mobilisables pour des actions collectives. Quant aux projets de développement, par contre, leurs modes d'interventions relativement standardisés et leurs effets relativement faibles n'ont rien de spécifique au pays. On retrouve au Niger comme ailleurs les opérations préférentiellement "communautaires", la multiplicité des intervenants, leur méconnaissance des arènes locales, la création d'un "espace de soupçon", et des capacités décevantes d'enclenchement d'innovations durables. Au carrefour de ces deux séries de phénomènes, on constate l'absence d"'espace public" et de "bien public".

3 Les enquêtes qui mènent à de telles conclusions ont porté sur quatre sites. Deux sont situés dans le centre-est du pays (région de Maradi), à savoir d'une part Shadakori, une grosse agglomération composée de plusieurs quartiers-villages, chef lieu de canton, et d'autre part un village du même canton, de taille plus modeste, ln Wala Tarnroro, situé à quelques kilomètres. Dans la région de Gaya, les deux villages choisis ont été d'une part Bana, chef lieu de canton, et d'autre part un ensemble de trois villages mitoyens proches du fleuve, Albarkaïze, Hima Koira et Tungagumbi ${ }^{1}$. De ce fait, la base empirique de nos analyses reste, au sens strict, étroite. Ces quatre sites enquêtés ne recouvrent pas toutes les régions du Niger, et donc ne reflètent qu'une partie des configurations politiques, sociales et culturelles existant dans le pays.

Cependant, la méthode ECRIS utilisée ${ }^{2}$ permet de compenser une partie de ces handicaps en proposant une analyse plus fine et détaillée que les enquêtes de types "survey" ou "enquêtes rapides" qui sont courantes dans le "monde du développement", et prend en compte systématiquement les aspects comparatifs négligés par les études monographiques classiques d'inspiration socio-anthropologique. En outre, la connaissance que l'ensemble de l'équipe ${ }^{3}$ avait déjà acquise du monde rural nigérien au 
fil de nombreuses enquêtes antérieures permet de procéder à quelques généralisations (qu'il faudrait toutefois faire précéder de la clause restrictive "sous réserve de recherches complémentaires"). Une grande partie des analyses ici proposées s'applique, pensons-nous, à la majorité des villages nigériens (plus précisément ceux qui sont situés dans l'arc agricole compris entre la frontière nigériane au Sud et les zones de pluviométrie à hauts risques au Nord).

La chefferie et l'espace politique local La chefferie au Niger

En Afrique de l'Ouest, le Niger est le seul pays, avec le Togo, à s'appuyer encore sur l'institution de la "chefferie", d'héritage colonial, comme seul mode d'exercice du pouvoir local. En dehors de quelques communes urbaines (dont les maires sont d'ailleurs nommés et non élus) le monde rural ne connait ni municipalités ni maires, ou leurs équivalents ${ }^{4}$. Les chefs de village sont actuellement élus à vie (sauf révocation) par les villageois, parmi des candidats qui doivent tous avoir en principe un "droit" légitime à prétendre à la chefferie, autrement dit être des descendants en ligne patrilinéaire des anciens chefs du village (chefs de cantons et sous-préfets décident de la légitimité des candidatures). La chefferie de canton, qui "coiffe" les chefs de villages, constitue en fait le principal centre de pouvoir officiel en milieu rural, et son rôle politique et symbolique est très important. Les chefs de canton, désormais presque tous d'anciens cadres de la fonction publique, sont élus à vie (parmi les descendants en ligne patrilinéaire des anciens chefs du canton) par les chefs des villages que compte le canton (les cantons, de taille variable, peuvent regrouper jusqu'à plusieurs dizaines de villages) ${ }^{5}$.

6 Les chefs de village (qui n'ont d'autre rétribution qu'une "remise" sur le montant de l'impôt qu'ils récoltent et remettent au chef de canton) et les chefs de canton (qui reçoivent, outre une remise sur l'impôt, une allocation annuelle, variable selon leur "indice") dépendent directement du Ministre de l'intérieur. Celui-ci peut les révoquer et provoquer une nouvelle élection. Ce sont donc, comme à l'époque coloniale, avant tout des auxiliaires de l'administration, même s'ils sont considérés aussi comme des représentants des populations, rôle que, pour une part, ils jouent. Les cantons eux-mêmes sont des créations coloniales, qui parfois recoupent en partie des réalités politiques locales pré-coloniales, parfois ont été créés de toutes pièces, et dont les limites ont été souvent modifiées sous la colonisation et depuis l'indépendance. La chefferie au Niger est donc une "chefferie administrative", d'origine coloniale, qui perdure depuis l'indépendance, et qu'il ne faut pas confondre avec ce qu'étaient les chefferies pré-coloniales ${ }^{6}$.

7 Mais cette "chefferie administrative" se revêt elle-même, et est revêtue par les autorités, d'une "légitimité traditionnelle", supposée être d'essence pré-coloniale. On parle d'ailleurs quasi-systématiquement de "chefs traditionnels" à propos des chefs en place. L'association qui les regroupe s'appelle "association des chefs traditionnels du Niger". Tout un apparat et toute une idéologie produisent et reproduisent cette légitimité soi-disant "traditionnelle", avec diverses "inventions de tradition" (ou néo-traditions) et le recyclage de certains éléments des rituels politiques pré-coloniaux, insérés dans un contexte tout différent ${ }^{7}$.

8 Une réalité sociologique permet cet usage légitimant de la "tradition" : c'est le fait que les chefs soient presque partout issus de l'aristocratie en place à la veille de la conquête coloniale. Le fait qu'ils soient de plus en plus souvent d'anciens hauts fonctionnaires ne change rien à l'affaire. En effet, les "grandes familles" du Niger ${ }^{8}$ associent en général 
des droits à la "chefferie" (et sont donc partie prenante de l'aristocratie), un accaparement des hautes fonctions dans l'administration, et un investissement dans la classe politique et le monde des affaires. La chefferie de canton, qui n'est qu'une des cordes à leur arc, est cependant l'objet de fortes convoitises. Elle est un enjeu important pour la "nomenclatura" nigérienne, et les intrigues qui précèdent l'élection d'un nouveau chef de canton sont une affaire d'Etat. L'association des chefs traditionnels du Niger est un lobby national puissant. Les chefs ont été et sont encore "courtisés" par certaines forces politiques (en particulier le MNSD autrefois, le régime actuel maintenant).

Et pourtant la vie politique récente semble indiquer que l'influence politique "de masse" de la chefferie est loin d'être aussi forte que l'on aurait pu le croire. Le temps n'est plus, s'il a jamais existé ${ }^{9}$, où les populations suivaient fidèlement les consignes de vote de la chefferie : les dernières élections présidentielles en témoignent.

La chefferie de canton comme enjeu local

10 Si la chefferie est un enjeu à l'échelle nationale, c'en est bien évidemment un aussi, et surtout, à l'échelle locale. La chefferie de canton est l'instance centrale de la notabilité locale. En témoignent de multiples signes: les courtisans qui entourent le chef de canton, les gardes personnels que, souvent, il entretient, les titres qu'il distribue ${ }^{10}$, les audiences qu'il accorde, les solliciteurs qui se pressent, le cérémonial qui le met en scène. C'est par lui ou grâce à sa médiation que, pour une part, la reconnaissance sociale s'évalue, ou que la réputation se jauge. Sur une seule personne ou une seule fonction se concentrent ainsi une grande partie des attentes des élites locales.

11 Mais cette visibilité et cette centralité du chef de canton a son revers de médaille : elle se paye d'innombrables conflits et intrigues, en particulier au sein du clan des "ayant-droit". Les candidats malheureux, les parents évincés, les cousins désavoués ne ménagent pas aigreurs et rumeurs. Nulle part mieux que dans les familles de chef on comprend pourquoi l'expression "enfants d'un même père" (baabizey en zarma, yan uba en hausa) connote partout au Niger la jalousie et la rivalité. Il n'est guère de chefferie de canton sans coterie contre le chef en place.

12 La concentration des signes du pouvoir local sur une seule personne, et le monopole de la fonction par les différentes branches d'une seule famille convergent pour rendre compte de la permanence et de l'acuité des conflits autour de la chefferie de canton. Mais il faut aussi ajouter qu'il y a toujours plusieurs micro-légitimités qui s'affrontent. Il ne s'agit pas simplement de vainqueurs et de vaincus à l'intérieur d'une procédure claire de désignation, mais d'affrontements sur les règles même du jeu, qui sont en fait multiples et non stabilisées. Tout le problème est en effet de savoir qui est "ayant-droit". Parmi les prétendants, chacun veut obtenir la disqualification de ses adversaires et sa propre qualification. Depuis les chefferies pré-coloniales, dont les chefferies coloniales et post-coloniales se proclament à bon ou mauvais droit les héritières, les nominations et dépositions de chefs, dans des branches rivales, se sont partout succédées, au gré de l'arbitraire et des préférences du pouvoir central, celui des gouverneurs coloniaux d'abord, celui des ministres de l'intérieur et chefs d'Etat ensuite, multipliant ainsi les contentieux et les revendications entre les divers héritiers de ces chefs successifs. De plus, les limites des cantons ont elles aussi varié, excluant ou incluant par là même telle ou telle fraction de l'aristocratie locale du groupe des prétendants. Tout projet de redécoupage des cantons devient automatiquement une affaire nationale, du fait de ses conséquences pour les familles de chefs et de 
prétendants. On ne s'étonnera donc pas que nous ayions rencontré dans les agglomérations où nous avons enquêté les mêmes conflits et divisions en factions autour de la chefferie que dans tout le Niger rural.

Un mot sur les chefs de village. On a en fait deux types de chefs de village : celui qui se contente d'être un arbitre, voire un simple nom sur un registre, et celui qui "fait le chef" et tente d'agir en miniature comme un chef de canton, avec moins d'autorité, moins de cérémonial, moins de compétences et moins de moyens. Mais il y a cependant une nette différence qualitative, marquée en particulier par la différence de titres et de considération (amiiru ou sarki pour les chefs de canton, koyra koy ou maïgari pour les chefs de village, en zarma et en hausa), et par le fait que les chefs de canton sont les supérieurs directs des chefs de village, et que eux seuls maîtrisent les rapports avec l'administration et la capitale. Quant à la transformation d'un ancien "quartier" (dépendant d'une chefferie de village située ailleurs) en village administratif reconnu (avec sa propre chefferie, ce qui suppose l'accord des autorités préfectorales et du chef de canton), c'est une source inépuisable d'intrigues locales, liée à l'enjeu que cela représente pour la famille prétendant à cette nouvelle chefferie. La chefferie de village, suscite les mêmes querelles et les mêmes dépits, à plus petite échelle, que la chefferie de canton.

Force de la chefferie de canton

14 Sa force réside dans ses fonctions reconnues d'arbitrage et de conciliation (de "justice de paix") d'un côté, mais aussi, et à travers elles, dans sa capacité de nuisance et de prélèvements. L'hostilité d'un chef de canton peut rendre la vie difficile, et ses administrés ne se permettent guère de l'affronter directement. Ses pouvoirs délégués en terme d'administration, de justice et de police sont à la base de ce constat. A l'échelle locale, il n'y a aucune "divisons des pouvoirs". Le chef de canton cumule en sa personne tous les pouvoirs : il représente le préfet, il perçoit l'impôt, il juge, il réprime. Et, à travers ces diverses fonctions, il prélève.

La nouvelle génération de chefs de canton, issus de l'administration (depuis l'ancien enseignant ou ancien infirmier jusqu'à l'ancien ministre), qui souvent maîtrisent des réseaux influents dans la capitale, est même en position de force face aux sous-préfets, dont théoriquement ils dépendent. Les cas ne sont pas rares, et nous en avons rencontré, d'un sous-préfet démis parce qu'il déplaisait à un de ses chefs de canton. Pour un villageois, saisir un sous-préfet contre un chef de canton est une entreprise à haut risque.

Par contre le chef peut faire arrêter quelqu'un par ses "cavaliers", et le faire conduire à la gendarmerie.

Le chef de canton juge en appel des chefs de villages, pour toute une série de conflits et contestations, pour l'essentiel des litiges fonciers, des querelles d'héritage, des affaires de divorce ou d'adultère, des bagarres entre individus, des larcins, des conflits entre éleveurs et agriculteurs. Il applique, le plus souvent sans aide d'un "cadi", un mélange de droit coutumier et de droit islamique, aux frontières imprécises, avec une large marge d'évaluation personnelle. Outre un "droit de table" parfois exigé (cette somme forfaitaire versée par les deux parties en conflit est une héritière du droit colonial), divers autres prélèvements plus ou moins occultes alimentent, grâce à la justice, la cassette du chef de canton ou de ses hommes de main: "droit de convocation", amendes infligées et perçues directement, commissions sur les dommages et intérêts, 
et sommes très souvent versées en sous-main pour se gagner la bienveillance du chef ou de ses conseillers :

Eh outre, le chef bénéficie de prestations ou de tributs "traditionnels": séances de travail collectifs des jeunes sur ses champs (bogu en zarma, gaya en hausa), bottes de mil offertes à la récolte, contributions en nourriture ou en travail lors des réceptions ou fêtes, dons permanents des solliciteurs. Certes le mélange d'exactions et de répressions, si caractéristique de la chefferie aux débuts de l'époque coloniale ${ }^{11}$, a disparu, et l'ampleur des ponctions a très nettement diminué. Les chefs ne peuvent plus réclamer la "dîme" (zakkat) sur les récoltes et prendre comme bon leur semble les animaux de leurs sujets. Mais la "pression morale" n'a pas disparu, et les contributions "volontaires" des villageois restent non négligeables. La marge d'arbitraire ou de despotisme que détient le chef de canton, à travers ses fonctions de justice et de police, bien que nettement moindre aujourd'hui qu'hier, rend ses sujets enclins à le ménager.

Il faut dire aussi que le chef de canton a un "rang à tenir" et que sa charge lui impose de nombreuses dépenses, dont beaucoup relèvent de la redistribution. Aumônes et cadeaux, entretien des émissaires, voyages et tournées, réception des visiteurs, le "budget" de la chefferie est lourd. Le problème est qu'il n'y a justement pas de budget. La chefferie de canton n'a aucun budget de fonctionnement, malgré quelques indemnités de logement ou de déplacement rarement versées, et n'est donc tenue à aucune comptabilité. C'est en quelque sorte de façon institutionnelle et légale qu'il y a complète confusion entre dépenses de fonction et dépenses personnelles, ce qui est la définition même du "patrimonialisme". Le pouvoir en milieu rural nigérien, bien que partie intégrante du système administratif moderne post-colonial, est donc très officiellement patrimonial, à l'image du sultanat classique pris comme exemple par Max Weber ${ }^{12}$. Le chef doit se "débrouiller" pour trouver les ressources qui lui sont nécessaires, et celles-ci lui permettront indissociablement et simultanément d'assurer les charges liées à sa fonction, de maintenir ou d'accroître son prestige, et d'augmenter son patrimoine personnel. On en verra les effets à propos des projets.

Il faut aussi signaler que le chef entretient tout un réseau de clientèle, dont il est très difficile d'évaluer les flux économiques et monétaires qu'il engendre. De même les chefs de canton ont souvent d'importantes ressources propres, soit en raison de la constitution d'un patrimoine foncier important (accumulé ou accaparé par la chefferie au fil des années) soit parce que, en général à travers leurs familles, ils sont impliqués dans le commerce, à une échelle parfois importante.

Le patrimonialisme officiel de la chefferie est certes distinct du "néo-patrimonialisme" qui s'est mis en place depuis l'indépendance aux postes de responsabilité de l'Etat ${ }^{13}$ (et qui semble s'être accentué depuis la "conférence nationale" qui a inauguré la démocratisation), dans la mesure où ce dernier est, lui, une "norme pratique" en contradiction avec les normes officielles qui régissent le fonctionnement de l'Etat. Les prébendes, avantages de fonctions excessifs, abus de biens sociaux, usages privatifs de fonds secrets, pratiques corrompues, commissions indues et autres prélèvements illicites opérés par les plus hautes autorités du pays n'ont aucune légitimité officielle : cette "culture politique" post-coloniale ne fonctionne que grâce à un détournement des institutions et un déni du droit ${ }^{14}$. Mais on peut émettre l'hypothèse qu'elle acquiert une légitimité "officieuse" du coté de la chefferie de canton. Autrement dit, la culture politique de la chefferie de canton est au coeur de la culture politique nigérienne, et ses procédés 
et son idéologie latente s'étendent de façon masquée aux institutions modernes, y compris et surtout le sommet de l'Etat ou les partis politiques...

Faiblesse de la chefferie de canton

Tout d'abord, il faut constater que le pouvoir du chef de canton est nettement plus prégnant dans le village où il réside que dans les autres. C'est là où se trouve sa cour que son autorité s'exerce le plus directement, que ses prélèvements sont de loin les plus manifestes, et le chef de village, nécessairement un de ses parents cadets, n'est alors qu'un figurant ou un auxiliaire. Par contre, dans les autres villages, son influence se fait beaucoup plus diffuse. Ses tournées restent rares, on ne se rend auprès de lui qu'en cas d'affaire à traiter ou de jugement porté en appel, et la fonction de chef de village s'interpose entre les paysans et le chef de canton.

Mais surtout le chef de canton n'a guère de capacités d'animation ou d'impulsion collective, pour les cas que nous avons eu à connaître, et même lorsqu'il est craint ou respecté. S'il peut nuire, s'il peut prélever, il ne peut guère ordonner ou entraîner, en tout cas pas dans les domaines qui relèvent de la vie sociale ou économique quotidienne, ceux-là mêmes où interviennent les opérateurs de développement. Regrouper les jeunes, les femmes, la population pour des tâches d'intérêt collectif ponctuelles est pour lui déjà difficile, mais pour une activité régulière ou sur le long terme c'est une mission quasi impossible : il n'est à cet égard simplement pas suivi, pas écouté. Quand bien même il s'y efforce, il n'arrive guère à faire assurer l'entretien du village, le nettoyage des espaces publics, la maintenance des puits. Il n'a, pourrait-on dire, ni légitimité "municipale", ni légitimité "mobilisatrice".

Il n'a pas de légitimité "municipale" parce que l'émergence de cette notion -inexistante pour le moment- est en fait incompatible avec l'institution de la chefferie sous sa forme actuelle. La personnalisation du pouvoir, sa patrimonialisation, son accaparement par une famille et sa concentration sur le seul chef de canton, tout cela n'est guère propice au développement d'une conception partagée d'un patrimoine collectif et d'un espace public géré en commun. Un village nigérien est un assemblage d'individus et de collectifs particuliers, ce n'est en rien une "commune" dotée d'infrastructures appartenant à tous et dont les responsables doivent rendre des comptes à leurs mandants. Soixante années de colonisation et près de quarante années d'indépendance ont au contraire développé des habitudes diamétralement opposées. Le chef de canton, seule autorité véritable et reconnue en milieu rural, n'est donc pas perçu par ses sujets comme représentant un "bien public", en fait inexistant. Ceux-ci ne se sentent donc pas tenus de le suivre s'il invoque l'intérêt collectif. Les marques de respect public dont il fait l'objet, le cérémonial traditionnel dont il s'entoure, l'autorité "naturelle " qui semble de dégager de lui ne doivent pas abuser : les populations qu'il administre ne le considèrent pas (au moins d'après notre expérience, nos observations et nos entretiens) comme l'émanation d'un intérêt général et le gérant d'un "espace public" commun.. L'institution de la chefferie est manifestement incapable de régler cette question de la maintenance des infrastructures "collectives", considérées comme n'appartenant à personne, même si leur usage est censé profiter à tous.

Le chef de canton n'a pas non plus de légitimité "mobilisatrice". D'autres institutions en ont pourtant eu, dans le passé, en milieu rural, au moins pour un temps : sans doute le RDA, sous le régime de Diori Hamani, mais surtout la Samaria, sous le régime de Seyni Kountché. A l'époque, le "samari" (responsable de la samaria, organisation de la jeunesse) était une figure politique présente dans absolument tous les villages, aussi 
importante que le chef de village, justement en raison de cette capacité mobilisatrice, qui s'était développée en quelque sorte comme une fonction nouvelle et autonome, en dehors de la sphère de compétence des chefs. Cette capacité mobilisatrice venait "d'enhaut" : c'était le produit d'un appareil puissant de propagande autour d'un leader national charismatique et autoritaire. La meilleure comparaison, c'est sans doute avec les "partis uniques" du monde communiste (bien que l'idéologie des deux régimes nigériens successifs ait été toute autre) qu'il faudrait la faire : comme eux, le PPN-RDA puis la samaria "encadraient" la population, et comme eux ils l"'organisaient" à l'échelle locale, créant par là même du "tissu social" apte à effectuer des tâches collectives. Certes la peur de la répression n'était pas absente. Mais elle ne peut à elle seule expliquer les multiples activités développées en particulier par les samaria: travaux collectifs, entretien des villages, construction partout d'un local ad-hoc, fêtes, compétitions ou invitations entre villages. Ces formes de mobilisation, qui n'existent plus depuis la démocratisation, n'ont jamais été le fait de la chefferie, qui est bien incapable de les récupérer.

Signalons une conséquence de cette faiblesse de la chefferie. Si les chefs de la nouvelle génération, qui sont d'anciens cadres, parlent désormais volontiers de "développement", et tentent de séduire les "projets", leur compétence n'est au fond que rhétorique, dans la mesure où ils n'ont guère de capacités d'animation ou d'organisation d'actions collectives en ce domaine (sauf exception, que nous n'avons pas rencontrée).

L'arène politique locale

Celle-ci est en fait fort peu structurée. En dehors de l'institution de la chefferie, ce qui inclut à la fois le chef en place et les intrigues de ses concurrents passés ou futurs, les autres institutions sont quasi inexistantes, ou n'interviennent pas dans la vie collective. Mais bien sûr opinions, débats, critiques, et rumeurs vont bon train.

Nous venons d'évoquer la disparition ou l'étiolement des samaria. Si parfois l'institution existe encore, elle n'est plus active, ou se réduit à un groupe informel de jeunes comme il en est partout. Les associations des femmes, autrefois généralisées dans la foulée des "mouvements de masse", sont de même aujourd'hui totalement léthargiques.

Les partis politiques, dont on a pourtant pu constater la surprenante implantation dans les campagnes dès qu'il s'agit d'élections nationales, n'interviennent en fait jamais sur la scène villageoise proprement dite. Certes nombreux sont ceux, dans les villages, qui se disent du parti X ou Y (les affilations peuvent changer au fil des années). Mais cela semble réservé aux échéances électorales, ou au soutien verbal à tel ou tel leader national, sans conséquence aucune pour les affaires locales ${ }^{15}$, ni pour les relations quotidiennes. Dans les seuls cas où on a pu constater que le clivage partidaire avait été invoqué dans un conflit villageois, ce clivage partidaire ne faisait que reproduire un autre clivage (entre quartiers ou entre factions : la faction A ayant choisi le parti X, la faction B a choisi le parti Y) clairement à l'origine du conflit.

L'islam, de son côté, n'intervient guère en tant que tel dans l'arène politique villageoise. Certes le rôle de l'imam est important, c'est un notable villageois ${ }^{16}$, et il peut être consulté, mais sa fonction est considérée comme uniquement religieuse, et elle n'induit pas des formes d'organisation ou d'animation amenant les clercs ou les fidèles à intervenir directement, au nom de l'islam, dans les affaires villageoises courantes, à la différence de ce que font ailleurs les églises chrétiennes (ou de ce que font, ailleurs également, les islamistes). Parfois l'imam est partie prenante dans une 
faction locale avec la chefferie pour enjeu, mais c'est en ce cas à titre personnel et en fonction de sa position dans les rapports de parenté qu'il agit. L'association islamique, quand il y en a une, reste sans activités dans les villages, et n'intervient pas non plus hors des questions religieuses. Certes les progrès de la confrérie fondamentaliste et puritaine "isaliste" déclenchent souvent, là où elle s'implante, de violents conflits dus à son intransigeance. Mais ces conflits restent eux aussi cantonnés au domaine religieux (en particulier autour de questions de rites, de mosquées ou de cimetières), sans se mêler vraiment des autres problèmes de la cité ${ }^{17}$.

Enfin les guérisseurs et magiciens, ou les confréries des cultes de possession (holley, bori), souvent marginalisés en milieu rural par la montée du rigorisme islamique, se limitent à leurs pratiques thérapeutiques et/ou rituelles, et ne jouent plus à l'échelle du village qu'un rôle relativement mineur, et surtout sans fonctions politiques: cérémonies pour réclamer la pluie, ouverture de la saison de pêche... ${ }^{18}$

Le tableau d'ensemble est donc paradoxal : on pourrait parler, malgré (et, en fait, à cause de) cette institution centrale et centralisée qu'est la chefferie, d'une très faible structuration de la société politique locale, et même, au sens où on l'entend en Europe, de la "société civile" villageoise, au moins en ses aspects organisés et intervenant dans la vie publique ${ }^{19}$. Autour de la chefferie, à la fois forte et faible, empêtrée dans ses querelles dynastiques, c'est au fond le vide institutionnel et politique. Tout se passe donc dans l'informel, les rapports inter-personnels, les "réseaux invisibles".

Le profond "individualisme" des paysans ${ }^{20}$, souvent déploré par les agents de développement, est à mettre en rapport avec ce constat.

Les projets au villageUne distribution éphémère de ressources par des étrangers multiples

On peut par commodité distinguer deux types de projets : (a) ceux qui passent par les services techniques officiels et constituent des projets d'appui aux agents de l'agriculture ou de l'élevage, aux instituteurs, ou aux infirmiers en poste dans les villages, projets dont en général les villageois ne connaissent pas l'existence (PRSAA pour l'agriculture et l'élevage, PROSEF pour l'enseignement, Initiative de Bamako pour la santé), et (b) ceux qui ont une existence autonome à l'échelle locale, intervenant directement avec leur propres agents, et connus en tant que tels par les villageois (Care, PAIGLR, CLUSA, etc.).

35 La première caractéristique de la présence des projets dans les villages est leur multiplicité et leur dispersion. Dans les zones de Maradi et de Gaya, il n'est sans doute aucun village qui n'ait connu au minimum plusieurs projets successifs, et nombre d'agglomérations voient aujourd'hui l'intervention simultanée de plusieurs projets, en général sans coordination entre eux ${ }^{21}$. Cet éclatement des opérateurs va de pair avec le sentiment que chaque projet se croit le seul, et ne tient guère compte de l'existence d'une histoire locale des interventions précédentes (par d'autres projets ou par les services techniques), qui marque pourtant profondément la perception que les paysans ont des projets actuels. Chaque projet, en outre, tend à se comporter en institution autonome et souveraine, étanche par rapport aux autres projets et par rapport à l'administration locale.

Un retour en arrière montre en fait la faible durée de vie des projets, et à quel point ils représentent pour les villageois des "étrangers qui ne font que passer" : trois ou quatre années d'activité représentent déjà pour un projet une "longue" présence. Mais la fin d'un projet ne signifie pas qu'il s'en aille parce que les conditions de la reproduction de 
ses activités sur une base purement locale ("durabilité") seraient réunies : au contraire, la disparition d'un projet entraîne à peu près systématiquement la disparition des activités qu'il impulsait.

On a donc la séquence paradoxale suivante : tout projet se veut transitoire, et créant les conditions de son appropriation et donc de son départ (stratégie officielle); tout projet se comporte en fait comme une institution recherchant sa propre perpétuation (stratégie de fait) ; tout projet plie un jour bagage subitement, et tout s'arrête alors, ce qui signe l'échec à la fois de la stratégie officielle et de la stratégie de fait. L'orientation "communautaire" ou "communautariste" des projets au niveau des villages doit aussi être mentionnée. A peu près tous passent par la création de "comités", de "bureaux", de "coopératives", d"'associations", qui veulent être aussi représentatifs que possible des populations. Ces "comités", pour la création et la vie desquels les agents de développement dépensent beaucoup d'énergie, et qui apparaissent pour les populations comme des "tickets d'accès" aux ressources des projets, semblent correspondre à plusieurs logiques différentes dans l'esprit de leurs initiateurs, logiques parfois dissociées, parfois cumulées :

- une logique "d'apprentissage démocratique" : ces comités doivent créer des habitudes démocratiques dans la population locale (élire des représentants qui gèrent des ressources communes et doivent rendre des comptes) ;

- une logique de "renforcement des faibles" (empowerment) : ces comités doivent permettre aux groupes marginalisés (femmes, jeunes, pauvres) de se renforcer ;

- une logique de "création d'interlocuteurs" : ces comités doivent permettre aux agents du projet d'avoir des "relais villageois" assurant l'interface entre le projet et l'ensemble de la population ;

39 Ces diverses logiques sont plus contradictoires qu'il n'y parait, et le succès de l'une se fait souvent aux dépens des autres. Ainsi les responsables des comités, en qui tel projet voit volontiers des "représentants" démocratiquement désignés de la population, apparaissent souvent pour les autres paysans comme "les hommes" du projet, qui "mangent" grâce au projet. Ou bien encore l'élection des responsables aboutit le plus souvent à mettre des notables aux postes de commandes.

On peut aussi estimer que la multiplication des "comités" par les projets relève d'une stratégie plus ou moins consciente de pérennisation-appropriation: après le départ futur du projet, sa pérennisation serait assurée essentiellement par l'existence de telles structures, leur bon fonctionnement pendant la période de tutelle des agents du projet apparaissant comme un indicateur d'appropriation. Cette stratégie semble démentie par les faits : au cours des trente dernières années, la fin d'un projet a entraîné quasi systématiquement la disparition des "comités" qu'il avait mis en place ${ }^{22}$. On reparlera en conclusion du rapport entre "comités" et espace public.

41 Enfin, il faut souligner la méconnaissance profonde que la plupart de ces projets ont des mécanismes réels des sociétés locales, et des stratégies que les acteurs villageois déploient à leur égard (mais il y a bien sûr des exceptions). On pourrait parler d'un certain "amateurisme", ou d'une "incompétence" de nombreux projets, non pas tant sur le plan "technique" que par rapport aux contextes sociaux et aux comportements des paysans, les opérateurs semblant, malgré le discours "participationniste" aujourd'hui de rigueur, souvent peu au fait du fonctionnement quotidien d'un village nigérien, avec ses quartiers, ses groupes, ses factions, ses réseaux, sa chefferie, ses querelles, ses soupçons...23 
42 Et pourtant un certain nombre d'agents de développement de base connaissent bien le milieu rural et le "terrain", et ne sont eux-mêmes ni vraiment dupes ni vraiment naïs dans leurs relations inter-personnelles avec les paysans. On a parfois l'impression que le fait de travailler dans le cadre d'un projet les fait entrer dans une sorte de schizophrénie, le volontarisme du projet modelant leur attitude professionnelle et officielle, sans communication avec leur expérience personnelle du monde rural qui ne s'investit que dans leurs jugements privés.

Des thèmes d'intervention récurrents débouchant sur quelques innovations adoptées

43 Au fil des années, on est frappé de voir que les actions de développement menées dans les villages, comme les thèmes sur lesquels les intervenants (services techniques ou projets) tentent de susciter des innovations, sont très largement identiques, même si les discours d'accompagnement ou les méthodes ont pour une part changé. Il faut se rappeler d'ailleurs qu'au Niger le service de l'animation rurale, dès le début des années 1960 et jusqu'au coup d'Etat militaire de 1973, a été un précurseur diffusant à peu près tous les thèmes aujourd'hui en vogue, de l'alphabétisation aux matrones, ceci dans une perspective déjà très "participationniste".

44 On peut faire facilement l'inventaire des principales actions de développement menées depuis plus de 30 ans dans les villages nigériens.

Le crédit

45 C'est devenu depuis quelques années un thème majeur, et sur les quatre sites l'essentiel des actions menées par les différents intervenants tournaient autour du crédit, sous presque toutes les formes possibles (de la tontine améliorée à la banque rurale, du crédit en nature au crédit monétaire). A l'exception des tontines améliorées, toutes les structures mises en place ont des problèmes de remboursement. Il faut savoir que les paysans ont depuis longtemps une expérience du crédit, et, pourrait-on dire, une expérience des stratégies de non-remboursement du crédit ou de détournement des objectifs des crédits. L'animation rurale, l'UNCC, les sociétés cotonnières et arachidières ont distribué partout des crédits depuis les années 1960. Bien souvent ceux qui ne les ont pas remboursé ont eu raison, puisque rares ont été les sanctions. On a de nombreux exemples de projets mettant fin subitement à leurs activités sans que les débiteurs, au moment de la fermeture, ne soient jamais inquiétés par la suite. Enfin, l'exemple vient d'en haut : il suffit d'évoquer la faillite de la BDRN en 1988...

46 Ne pas rembourser un crédit en espérant que le projet qui a distribué les crédits va fermer semble être une attitude qui ne manque pas de bon sens...

47 On a aussi de nombreux exemples de crédits dont l'usage a été "détourné" par leurs bénéficiaires tels ces crédits liés à l'achat de matériels agricoles qui ont été vendus peu après pour satisfaire des besoins sociaux exigeant du numéraire (mariage par exemple). On parlera plus loin de la "sur-monétarisation", cette pression constante pour trouver de l'argent liquide, qui constitue le contexte du "besoin de crédit" dans les campagnes comme à la ville.

Le fait que les attributions de crédits passent par des structures villageoises collectives créées ad hoc (comité ou bureau), ce qui est le mode de gestion exigé le plus souvent par les projets et les services techniques, ne simplifie pas les choses: les paysans responsables de cette gestion doivent ainsi jouer un rôle de banquiers-comptables-caissiers, sans en avoir les compétences, et tout en étant soumis à de fortes pressions sociales de l'entourage (qui rendent difficiles l'impartialité dans 
les attributions ou les sanctions contre les mauvais payeurs). Alors que les crédits sont utilisés individuellement, ce recours par les intervenants extérieurs à une notion de "responsabilité collective" (la "caution solidaire") à l'échelle du village (qu'ils imposent souvent au nom d'une stratégie de "participation communautaire" qui n'a guère d'existence au niveau local) ressemble étrangement aux usages de l'ancienne administration coloniale, qui ne voulait s'adresser qu'à des collectivités tenues comme solidairement responsables (ou coupables) ${ }^{24}$.

L'alphabétisation

C'est un autre axe d'intervention privilégié sur nos quatre sites, comme ailleurs dans le pays. C'est aussi un axe d'intervention qui date de plus de trente années. On a de ce fait l'impression d'un travail de Sisyphe, chaque projet recommençant son "volet" alphabétisation (ou parfois le recyclage d'anciens alphabétisés) sans que les campagnes précédentes n'aient laissé de traces. Il faudrait certes dresser un bilan plus fin et plus documenté, mais on doit constater que, en l'absence d'un "contexte lettré" en langues nationales, le peu de possibilités d'utilisation des compétences acquises transforme, pour un(e) paysan(ne), le fait de suivre pendant un an ou deux des cours du soir en un investissement personnel lourd, peu rentable, et, de plus, volatile. Seuls y ont vraiment trouvé leur compte quelques jeunes ayant pu être embauchés sur place, soit comme instructeurs en alphabétisation, soit comme gérants de caisses collectives, qui ont pu ainsi rentabiliser et entretenir leurs compétences.

D'un point de vue d'alphabétisation fonctionnelle, en particulier en ce qui concerne le calcul et la comptabilité, l'usage de l'écriture en langues nationales est très marginal. Soit les comptes restent oraux (cas de la plupart des tontines, même là où il y a depuis longtemps des cours d'alphabétisation), soit ils sont écrits en français par d'anciens écoliers de plus en plus nombreux dans les villages (voire, parfois, en arabe ${ }^{25}$ ).

Infrastructures collectives

51 Bâtiments, puits, pompes, construits par les projets ou les services techniques, sont censés être entretenus par la collectivité puisqu'ils ont une utilité collective. Mais qu'est-ce que la collectivité pour les sociétés villageoises ? L'absence de réponse claire à cette question fait que les problèmes de maintenance sont partout présents. La notion d'un patrimoine collectif villageois, et d'une responsabilité partagée à son égard, n'a rien d'évident. Il n'y a ni traditions ni habitudes ni formes endogènes d'organisation allant en ce sens. Un village nigérien est toujours une stratification et un chevauchement de groupes particuliers, et chacun soupçonne volontiers les autres de se défausser face à des responsabilités ou à des tâches "collectives".

En ce qui concerne les bâtiments, leur fonction première (boutique coopérative, banque de céréales...) disparaît en général avec les projets qui les avaient construits. Les bâtiments ne sont plus utilisés. La récupération par un autre projet ou l'abandon sont alors le sort qui leur est réservé.

Les pompes sont confrontées aux problèmes de financement des réparations. La vente de l'eau au seau pour constituer un fonds d'avance, ou la cotisation une fois la panne survenue pour réunir la somme nécessaire, sont les deux seules solutions, qui toutes deux soulèvent de nombreuses difficultés d'organisation, de mobilisation et de gestion à l'échelle du quartier en cause (cf. le chapitre sur la faiblesse de la chefferie) pour peu que les pannes se succèdent, qu'un détournement survienne ou que certains refusent la procédure choisie (ces trois cas de figure étant relativement fréquents). Les hommes ne 
sont peut-être pas non plus prêts à surmonter toutes sortes de difficultés pour simplement économiser la peine des femmes.

Seuls les puits cimentés semblent être entretenus normalement, dans la mesure où c'est l'accès même de tous à une ressource vitale qui est en question, et où il ne s'agit que d'un récurage, déjà pratiqué pour les puits dits "en bois" traditionnels ${ }^{26} \ldots$

Les agents de santé villageois

Du côté des "secouristes", matrones et, parfois, dépôts villageois de médicaments, plus de trente ans de tentatives réitérées ne permettent guère de présenter de bilan positif. Si l'implantation d'un dispensaire avec un infirmier suscite en général (mais pas toujours) une fréquentation forte, le problème se pose là où il n'yen a pas. Les formations brèves de paysan(ne)s pour effectuer des actes thérapeutiques simples et vendre quelques médicaments de premier secours n'ont jamais engendré de mécanismes auto-entretenus: les gestes s'oublient, les médicaments ne sont pas rachetés, les caisses sont détournées. La stratégie dite des "soins de santé primaire" a, sur le terrain, échoué. Aujourd'hui le recours à l'auto-médication et aux médicaments de colportage est massif dans les villages : c'est là que réside la véritable innovation, hors projets (hélas, d'un point de vue de santé publique elle est sûrement contestable).

Quant aux comités de santé villageois, ils n'ont d'existence que tenus à bout de bras par des projets ou par les infirmiers en poste.

L'Initiative de Bamako (IB), qui se fonde sur un recouvrement minimal des coûts, la vente de médicaments génériques, et la présence d'un gestionnaire, semble cependant avoir un impact réel : désormais on peut se fournir sur place, à des prix qui restent abordables, en médicaments de base. Il faudra cependant attendre la fin des interventions des projets qui soutiennent (en particulier en encadrant, contrôlant et subventionnant) la mise en place de l'IB pour évaluer la pérennisation du système.

Les techniques agricoles

58 En ce domaine, où il est vrai beaucoup d'énergie et de moyens ont été dépensés depuis 40 ans ${ }^{27}$, on peut noter désormais quelques acquis relativement récents, sans doute dus à la crise présente des systèmes de production, qui ne peuvent désormais plus fonctionner sur un modèle extensif. Ces innovations introduites et adoptées sont toutes mises en œuvre à l'échelle individuelle.

Ainsi le stockage de fumier au village et son épandage sur les champs avant l'hivernage sont des pratiques qui se sont généralisées ${ }^{28}$. Il est vrai que en ce domaine la première vague d'innovation introduite et adoptée avait été, dans les années 70 et 80, l'utilisation intensive d'engrais chimiques, à l'époque fortement subventionnés. Le coût actuel des intrants industriels et la baisse des rendements des sols ont incité les paysans à recourir désormais à cette technique de régénération non coûteuse et efficace qu'est la récupération des déjections humaines et animales ${ }^{29}$.

Mais cette innovation n'a été rendue possible que grâce à l'adoption préalable d'une autre innovation. La généralisation des charrettes (à traction bovine surtout, mais aussi asine) est sans doute l'innovation la plus spectaculaire et massive survenue dans les campagnes. Inconnues il y a 30 ans elles sont devenues un moyen de transport à tout faire désormais banalisé ; on peut y voir un "sous-produit ayant réussi" des actions en faveur de la culture attelée, qui, elle, a connu un succès plus inégal, entre autres parce que de nombreux types de sols s'y prêtent mal. 
61 D'autres innovations du même type semblent susciter un intérêt croissant en milieu rural, mais sont encore in d'être généralisées la confection de compost, ou l'enfouissement de fumier ciblé sur les poquets. Autre innovation qui semble se répandre, et qui correspond aussi à une prise de conscience de la dégradation des terroirs : le défrichement "amélioré", qui laisse sur le champ quelques jeunes arbustes ${ }^{30}$..

62 Enfin un certain niveau de protection des ressources naturelles s'est mis peu à peu en place ici ou là, mais, dans la mesure où il est assorti d'un côté d'une politique de répression des services officiels ("eaux et forêts") et d'un autre côté d'une injection de ressources par les projets, il est difficile de savoir si ce sont là des innovations qui ont véritablement "pris" (que se passerait-il s'il n'y avait plus ni répression ni projets ?) ${ }^{31}$. Il s'agit en particulier de la protection des rôneraies (interdiction totale de coupe de bois vif, et autorisation payante à demander pour le bois mort). Les coupes clandestines (parfois avec la complicité active de ceux qui sont censés les interdire) semblent cependant loin d'avoir disparu. Par contre, la mise en défens des mares de bas-fond (comme réserves de pêche) semble spontanément pratiquée par les riverains : il est vrai qu'il s'agit surtout là de protéger contre des pêcheurs amateurs venus d'autres villages une ressource locale future très circonscrite et limitée.

La promotion de spécialités locales

63 Les thèmes ci-dessus ont été promus et vulgarisés dans le pays tout entier, que ce soit par des projets autonomes ou par les services techniques, eux-même adossés à d'autres projets. Bien sûr on trouve aussi des thèmes particuliers, qui ont ici ou là été expérimentés, en particulier ceux qui sont étroitement liés à une "spécialité locale" que l'on tente d"'améliorer" techniquement : fabrication de miel épuré pour les apiculteurs, bacs d'évaporation solaire du sel pour les paludiers, filets et pirogues pour les pêcheurs, etc. En l'occurrence seul un bilan au cas par cas est possible. Dans tous les cas, il s'agit de secteurs caractérisés par une production faisant depuis longtemps l'objet de commercialisation et d'exportations "traditionnelles" parfois fort rentables (la pêche par exemple), où les innovations proposées s'appuient sur des circuits économiques déjà solides.

64 Le maraîchage (y inclus les vergers), bien qu'on ait tenté d'en faire une activité généralisée, promue à l'échelle de tout le pays, est devenu lui aussi une spécialité locale.

65 Il est très inégalement pratiqué. Initié "par en haut "sur une grande échelle lors du régime militaire (les "cultures de contre-saison"), il a souvent été abandonné, soit faute de débouchés commerciaux, soit parce que les sols s'y prêtaient mal. Dans certains endroits seulement il a "pris", et bien pris (comme à Bengou).

L'usage local des projets

66 Les effets des projets ne se résument pas à l'alternative adoption/rejet des propositions d'innovations. Il y a en effet toute une série d'effets sociaux et économiques induits, non attendus, indirects.

67 Parmi eux, l'importance des malversations (ou, en tout cas, des soupçons et accusations de détournement) doit être évoquée. Il n'y a aucun projet dans aucun village qui ne soit épargné, à tort ou à raison. Il semble que, même si les rumeurs sont parfois non fondées, l'existence de "trous dans la caisse" attestés soit quand même assez fréquente. Il y a manifestement un problème récurrent quant à la gestion de fonds communs (or 
on a vu que les projets exigeaient très généralement une structure collective de gestion). Pharmacies villageoises, boutiques coopératives, banques céréalières, coopératives de production et groupements divers sont ainsi régulièrement acculés à la fermeture du fait de l'indélicatesse de leurs gérants ou de leurs présidents. Ces pratiques sont beaucoup plus que l'exception, même si elles ne sont pas tout à fait la règle, et elles entretiennent un climat de soupçon permanent dans des sociétés villageoises déjà tissées de rivalités et de jalousies ${ }^{32}$.

La pression monétaire (sur-monétarisation), ou les faibles compétences locales en gestion et en contrôle de gestion figurent sûrement parmi les facteurs explicatifs de cette situation (la tentation de puiser dans la caisse pour tenir son rang socialement est d'autant plus forte que les contrôles sont lointains). Peut-être aussi l'absence d'une tradition de "bien public" et de distinction entre ressource commune et ressource personnelle (du haut en bas de la société) jouent-elles aussi un rôle. Les structures villageoises mises sur pied par les projets n'échappent pas vraiment au mode de gestion patrimonialiste, malgré les efforts de leurs promoteurs.

69 Un autre mode d'usage, ou d'appropriation (dans tous les sens du mot) des projets, consiste dans l'accaparement par un groupe ou une faction des ressources auxquels ils permettent d'accéder ${ }^{33}$. Là aussi le phénomène est généralisé, bien qu'il soit souvent masqué aux yeux des agents des projets et encore plus de leurs staffs, du fait de leur méconnaissance des relations de parenté ou d'affinité locales, voire parfois en raison du recours à des prête-noms. Les distributions de crédits par des comités normalement "élus" (et souvent en fait auto-désignés) dont les membres satisfont en priorité les demandes de leur propre famille ou de leurs proches sont ainsi une pratique courante.

Quant aux "commissions" et "cadeaux" donnés à ceux d'entre les villageois qui servent de "portes d'accès" aux ressources des projets ou qui les distribuent eux-mêmes, ces "prestations" sont à ce point d'usage courant qu'elles sont pratiquées spontanément, même lorsqu'elles ne sont pas formellement exigées. Ceci rejoint l'habitude courante de "remercier" qui vous a rendu service, et peu importe si c'est à titre personnel ou dans l'exercice de ses fonctions...

71 Le système des projets a d'autre part introduit un nouvel enjeu monétaire dans les villages : la question des "per-diem". Le fait que les agents de développement affectés dans les villages, qu'ils travaillent directement pour des projets ou que ce soient des agents de l'administration soutenus par des projets, fonctionnent de plus en plus au "per-diem" (ou, également, au "supplément de salaire" ${ }^{34}$ ) a fait tâche d'huile dans le monde paysan: de plus en plus les villageois réclament des "per-diem" ou des indemnités de la part des projets. Divers projets ont eux-mêmes contribué à introduire le problème directement dans les villages en versant des "per-diem" aux paysans en formation, non seulement à l'occasion de déplacements mais aussi lorsque les formations (en gestion, en technique, ou en alphabétisation) avaient lieu au village même. L'existence dé sonnais partout connue de cette "possibilité" se transforme vite en revendication d'un "droit", d'autant plus que chacun voit le "luxe" des projets (le véhicule tout-terrain climatisé flambant neuf avec son chauffeur en est le symbole), et que nombre de paysans pensent que c'est avec l'argent gagné grâce à leurs activités dans les villages que les projets peuvent entretenir un aussi haut niveau de vie.

72 Le principe du bénévolat est ainsi de plus en plus impossible à prêcher. De ce fait l'idéologie "participationniste" des projets, selon laquelle le travail (nécessairement gratuit) des villageois est un gage de leur intérêt, et constitue aussi leur apport propre, 
se heurte de plus en plus aux représentations et aux stratégies des populations. qui considèrent que les projets sont des moyens d'enrichissement pour leurs cadres et leurs agents sur le dos des populations, et qui estiment donc que eux aussi doivent avoir "leur part" en indemnités, per-diem ou rétributions directes diverses.

Chefs et projets

Dans tout cela, quels rôle jouent les chefs? On se rappelle qu'ils n'ont pas de véritable capacité d'animation et de mobilisation. Par contre, ils peuvent développer des stratégies d'accaparement ou de perception de commissions.

En fait on a pu constater trois "modèles" différents quant à l'attitude des chefs vis-à-vis des projets : l'ignorance, l'accaparement et le péage.

L'ignorance

Certains chefs (de moins en moins nombreux sans doute) se désintéressent des projets (voire y sont parfois hostiles en sous-main), dans la mesure où leurs enjeux sont ailleurs.

\section{L'accaparement}

C'est une stratégie que nous avons rencontrée plusieurs fois, et qui s'exprime par un noyautage assez systématique, sur une base familiale, des bureaux ou comités qui vont contrôler l'accès aux ressources apportées par les projets, et permettre ainsi de les drainer vers sa famille, son groupe ou sa clientèle.

Le cercle de drainage peut-être plus ou moins large. Dans certains cas, on aura une sorte de "main-basse" familiale sur les projets. dans d'autres cas, ce sera plutôt au bénéfice d'un quartier, voire d'un village, et donc au détriment des autres quartiers ou des autres villages. Quant aux hameaux périphériques (sans même parler des groupes doublement périphériques comme les Peuls), ils sont assez systématiquement marginalisés par rapport aux projets, ce que ces derniers semblent totalement ignorer, d'autant plus qu'ils se focalisent systématiquement sur "le village" - là où réside le chef - comme unité d'intervention.

Le péage

78 C'est là une stratégie à la fois de contrôle et de prélèvement. Sans s'immiscer directement dans les structures villageoises mises sur pied par les projets, le chef se contente de les surveiller et de jouer, plus ou moins directement, l'intermédiaire ou le facilitateur entre ses populations et les projets. Il en tire des bénéfices fort matériels du coté de ses sujets en recevant et souvent en exigeant "sa part" sur les avantages obtenus (crédits, subventions, matériels).

Fonctionnaires au village et projets

Dans les grosses agglomérations rurales (en général là où est la chefferie de canton), on trouve quelques fonctionnaires "affectés" : instituteurs surtout, puis infirmiers, parfois agents de l'agriculture, de l'élevage, de l'environnement, ou encore gendarmes. Certains sont appuyés par des projets, qui leur fournissent parfois une moto, parfois des per-diem ou compléments de salaires, et font ainsi figure de privilégiés par rapport à leurs collègues moins bien lotis (mais ce sont les agents directement employés par les projets qui sont les véritables privilégiés, leurs employeurs versant des salaires non seulement supérieurs mais encore réguliers, à la différence des salaires de la fonction publique). Ces projets d'appui sont toujours verticaux, à l'image des services et administrations d'appartenance. Au mieux, ils vont coordonner des services proches, 
comme le PRSAA, qui met en collaboration relative les agents de l'agriculture, de l'élevage et de l'environnement).

Quant aux projets intervenant directement dans les villages avec leur personnel, ils ignorent souvent les fonctionnaires en place. Quand il y a des exceptions, elles concernent comme indiqué ci-dessus des coordinations avec des agents de l'Etat intervenant dans le même domaine, par exemple le PAIGLR qui collabore selon les cas avec les agents de l'agriculture, de l'élevage ou de l'environnement.

81 Mais jamais un projet "crédit" ou "intrants", par exemple, ne tentera de s'appuyer également sur les instituteurs, les infirmiers, voire les gendarmes. Pourtant ceux-ci s'en plaignent très généralement, tant parce qu'ils ont le sentiment qu'ils sont ignorés ou que leurs compétences ne sont pas mises à contribution, que parce qu'ils souhaiteraient eux aussi bénéficier des avantages que procure le fait de travailler "avec" ou "pour" un projet.

Les contraintes "lourdes" de l'environnement socio-économique

82 Au total, chefs et projets nous apparaissent comme maîtrisant assez peu les principales forces qui modèlent en fait l'essentiel des comportements paysans actuels et d'où va sans doute surgir l'avenir de ces villages. Quelles sont ces forces?

Les échanges économiques

83 Dans les sites enquêtés, comme dans le reste du Niger, les forces économiques majeures échappent au contrôle tant des chefs que des projets. Il s'agit surtout des échanges commerciaux de divers types par lesquels transite désormais une part importante de la reproduction sociale des sociétés villageoises. Les prix de vente des produits agricoles et des intrants et les fluctuations des marchés, les flux migratoires, la contrebande avec le Nigéria sont des variables économiques majeures que personne ne maîtrise, ni même ne tente plus de maîtriser. Or ces divers circuits d'échanges génèrent aujourd'hui une grande part des revenus paysans. Si la part d'auto-consommation - qui évidemment échappe elle aussi complètement aux chefs et aux projets - reste importante, elle ne saurait faire oublier le rôle crucial aujourd'hui des échanges et de la circulation monétaire jusque dans les villages les plus reculés.

Il y a d'abord la commercialisation des produits agricoles, avec en premier lieu les cultures dites de "subsistance", mil et sorgho, qui sont devenues des cultures de rapport (vente des excédents); c'est encore plus évident avec le niébé, ou avec le souchet, vers Maradi (culture semble-t-il fort rentable, et qui s'est développée sans aucun appui des services ou des projets ${ }^{35}$ ), qui sont à divers égards des cultures spéculatives, ou encore, vers Gaya, avec le manioc ou le maïs. Ensuite, on a les cultures dites "commerciales" classiques, l'arachide encore, malgré son déclin, le coton dans la région de Gaya. La plus grande partie des ventes de tous ces produits agricoles de divers types se fait vers le Nigéria, en suivant des circuits "informels". De même, c'est au Nigéria qu'on s'approvisionne de plus en plus en intrants.

D'autres exportations à base de spécialités locales génèrent aussi des flux monétaires plus ou moins importants : nattes et cordes ici, sel du Fogha ailleurs, racines de pousses de doum (miriki) encore, ou bien, le long du fleuve, le poisson (source d'importants revenus malgré la baisse de la ressource : on parle de 20.000 à 50.000 CFA par jour pendant la saison de pêche).

Rappelons enfin l'importance du commerce du bétail. 
87 En outre, près de la frontière nigérianne, la contrebande devient une activité rurale centrale (essence en particulier). La naira est d'ailleurs dans ces villages la monnaie dominante. Ailleurs, c'est l'exode qui draine des flux réguliers d'argent vers les villages. purement agricoles, et sont en fait des pluri-actifs. Très nombreux d'ailleurs sont ceux et celles qui s'adonnent eux-mêmes au petit (et parfois moyen) commerce. activité que les projets ne prennent généralement guère en compte (pas plus que la contrebande, l'émigration ou la commercialisation).

Crise foncière

Le plus grand changement advenu dans les campagnes nigériennes depuis 40 ans est sans doute la saturation et la dégradation des terres cultivables. C'est un phénomène massif dont tous les paysans sont évidemment extrêmement conscients. L'ensemble du système traditionnel de cultures, basé sur l'existence de terres disponibles et les jachères de longue durée, s'est effondré ou s'effondre ${ }^{36}$. Il n'y a plus, dans cette bande du Niger agricole, ni terres disponibles, ni jachères de longue durée. On en a vu plus haut une conséquence en termes d'innovation, avec le recours d'abord aux engrais chimiques, puis, de plus en plus, au fumier. Mais est-ce là une solution à la mesure du problème?

Une autre conséquence en est la recrudescence partout inquiétante des conflits entre éleveurs et agriculteurs. Le rétrécissement des aires de pâturage et des couloirs de passage, grignotés sans cesse par les cultures, au moment où les troupeaux restent l'une des principales ressources nationales, entraîne une situation à laquelle on ne voit pas pour le moment d'issue. Les chefs sont débordés, et incapables de régler à long terme les problèmes, les quelques gendarmes à la disposition des sous-préfets arrivent à grand peine à ce que le nombre de morts d'hommes chaque année reste relativement faible, les tensions montent, les amphétamines et les armes circulent.

91 Ces conflits entre éleveurs et agriculteurs ne doivent pas être interprétés d'abord en termes ethniques ou comme des conflits "nomades/sédentaires". La plupart des Peuls, par exemple, sont des agro-pasteurs sédentarisés. Les paysans hausa, tchanga ou zarma sont aussi éleveurs ${ }^{37}$. Ces conflits renvoient en fait à une variété de situations, que l'on peut résumer par deux grands types de plaintes : du coté des bergers, on supporte de plus en plus malles difficultés croissantes de passage et d'abreuvement des troupeaux (soit les grands troupeaux transhumants, soit les troupeaux locaux) à travers des espaces autrefois pastoraux qui se réduisent sans cesse et sont de plus en plus gagnés par les champs; du coté des cultivateurs, on supporte de plus en plus mal les dégâts souvent considérables infligés aux cultures par les animaux. La complémentarité élevage/agriculture sur un même terroir en saison sèche (aire de pâture pour les uns, fumure des sols pour les autres) devient pendant l'hivernage une concurrence très conflictuelle entre des usages incompatibles d'un même espace. Les chefs arbitrent ces conflits au coup par coup, sans pouvoir s'attaquer aux causes, et sans pouvoir contenter jamais personne (éleveurs comme agriculteurs se plaignent systématiquement d'être désavantagés par les chefs, et les soupçonnent toujours de favoriser l'autre camp, ou d'avoir été "mieux" soudoyés par lui). Les projets évitent en général de s'affronter à ces problèmes, et se contentent les uns d'appuyer l'agriculture, les autres d'appuyer l'élevage, ce qui évidemment n'arrange rien. Quant aux services de l'environnement, ils rappellent les limites des couloirs et des aires, sans pouvoir faire grand chose quant à l'usage qui en est fait en pratique... 

foncière, et ceci d'autant plus qu'il y a une grande variété des normes d'usage, de prêt, d'appropriation et de location qui se sont empilées depuis le 19ème siècle, et l'époque où des terres vacantes étaient encore disponibles. Coexistent ainsi, en ouvrant la voie à toutes les contestations; les droits traditionnels sur la terre des premiers occupants, le prêt de terre sans délai de retour et sans redevance autre que symbolique, la mise à exécution par certains du slogan "la terre appartient à celui qui la travaille" proclamé par Kountché, les pratiques résiduelles de "mise en gage", un nouveau type de location à courte durée qui émerge, le développement spectaculaire des achats de terres (autrefois inconnus), le développement du partage des champs à la mort du père ou même bien avant (également autrefois inconnu), et l'expropriation des terres sans indemnité pour des aménagements hydro-agricoles, tout ceci en l'absence de cadastre et de titres de propriétés. Les difficultés innombrables que connait depuis dix ans la tentative de mettre en place un "code rural" sont un indicateur de l'ampleur du problème ${ }^{38}$, dont les approches dites "gestion de terroirs" sous-estiment souvent l'ampleur.

93 Enfin la question des arbres est à la fois une question de survie, une question foncière, et une question environnementale. Le bois reste dans les campagnes la seule source d'énergie domestique, et devient une ressource de plus en plus rare, que l'on doit chercher de plus en plus loin. Couper des arbres est donc une exigence impérative et permanente de la reproduction paysanne, et c'est parfois aussi une exigence de la production (couper les arbres de son champ reste le seul moyen de lutte connu contre les oiseaux prédateurs ; les feux de bois sont indispensables à la fumure des poissons ou à l'évaporation du sel ).

"Planter pour pouvoir couper" serait donc une stratégie logique, préconisée par certains paysans, mais qui se heurte tant à la politique de l'environnement (qui, dans les villages, est vécue comme "planter pour ne plus y toucher") qu'à la législation en vigueur, dans la mesure où les arbres sont censés appartenir à l'Etat, qui seul peut autoriser leur coupe, et non pas le propriétaire du champ où ils se trouvent, ni même celui qui les a plantés. On trouve ainsi les traces des "plantations villageoises" mises en place par Kountché à la suite de son "appel de Maradi" en 1984. Mais elles sont inexploitées ( $\mathrm{du}$ fait de l'interdiction générale de coupe), au grand dam des paysans, en particulier de ceux sur les champs desquels elles ont été faites... exploitation dans les campagnes est donc entier. Il devient chaque jour plus aigu (les foyers améliorés ne peuvent que l'atténuer sans le régler), et il n'est en général pas pris en compte par les projets forestiers ou énergétiques, qui se soucient surtout de l'approvisionnement des villes ou qui se concentrent sur des forêts classées ou des espèces protégées (comme les roniers ${ }^{39}$ )

Signalons enfin que, en ce qui concerne l'environnement, outre la déforestation, deux catastrophes naturelles sévissent actuellement face auxquelles Etat et projets sont complètement impuissants: la prolifération des jacinthes d'eau sur le fleuve, et la prolifération du Sida cordiphonia dans les couloirs de passage et aires de pâturage.

La sur-monétarisation

Il y a déjà 20 ans, Raynaut, au terme d'une étude fine d'anthropologie économique dans la région de Maradi, signalait l'importance de la circulation monétaire en milieu rural, et sa démesure par rapport aux gains monétaires proprement dits ${ }^{40}$ Un paysan qui

Bulletin de l'APAD, 15 | 1998 
aurait par exemple un revenu monétaire de $100.000 \mathrm{CF}$ A annuel verrait en fait circuler entre ses mains près d'un million CF A par an, au fil des dons et contre-dons, des achats et des ventes, des prêts et des restitutions, des échanges commerciaux, quotidiens, familiaux, sociaux. Il y a une constante pression pour du cash, une permanente quête de numéraire, qui ne diminue en rien à mesure que les gains augmentent: les fonctionnaires des villes en savent quelque chose. Plus on gagne d'argent, plus on est sollicité en de multiples occasions. "Tenir son rang", satisfaire à ses obligations sociales, cela suppose d'avoir sans cesse la main à la poche. Mariages ou baptêmes sont de bonnes illustrations, qui exigent, jusque dans les plus petits villages. des liquidités bien au-dessus des ressources habituellement disponibles d'un foyer. On connait par exemple les contraintes du système du biki hausa (chaque femme doit donner le double de ce qu'elle-même a reçu en une occasion similaire).

Il s'agit là d'une tendance lourde, qui n'est pas sans liens avec divers phénomènes qui étonnent ou scandalisent les coopérations étrangères c9mme la banalisation de la corruption. Elle constitue un contexte non pris en compte par les projets. malgré son importance. La demande permanente de crédit en milieu rural comme les détournements réguliers des caisses collectives y trouvent en partie leur origine (en partie seulement : une explication mono-causale n'est jamais vraie).

Le besoin de crédit, exprimé régulièrement par les paysans face aux projets, ne signifie pas un manque absolu d'argent, une absence de toutes ressources, un dénuement, mais l'insuffisance permanente des moyens monétaires de tout un chacun face à une pression sociale qui en demande sans cesse plus. De ce point de vue un paysan riche a encore plus besoin de crédits qu'un paysan pauvre...

La constitution d'une épargne en est sérieusement freinée. C'est pour cela que bien souvent seules les "épargnes forcées" peuvent se constituer, que ce soit Celle d'une tontine (grâce à la pression des voisines et du regard des autres) ou celle d'un crédit (si on est vraiment tenu de le rembourser).

101 En outre, les investissements productifs en agriculture dont le temps de retour est long sont loin d'être le meilleur "placement" (le commerce, la spéculation ou la contrebande sont nettement préférables, et permettent une bien meilleure circulation de l'argent). Cependant, pour peu qu'un "guichet" s'ouvre, et qu'un projet offre du crédit, il ne manquera pas de candidats, même si le crédit est conditionnel et lié à un investissement productif. Même si les taux pratiqués par ces "crédits de projet" sont finalement assez élevés, ils restent inférieurs aux taux usuraires pratiqués par certains commerçants. Le risque est que le matériel agricole ou le bétail acheté grâce à ce type de crédit soit revendu en cas d'urgent "besoin d'argent" (les ventes à pertes ou au plus bas des cours de céréales, de bétail ou de matériel ne sont évidemment pas liés à une quelconque ignorance des importantes fluctuations des cours ${ }^{41}$, mais à l'existence de telles "urgences" sociales).

ConclusionLes projets contre l'Etat?

102 Nous avons souligné la faiblesse relative, pour des raisons différentes, des chefs et des projets, et leur absence de contrôle sur les tendances socio-économiques lourdes dans les campagnes. Mais le grand absent, n'est-ce pas l'Etat, qui, un peu partout ailleurs dans le monde, réalise les infrastructures, promeut une politique économique et commerciale, régule le foncier, met en place et encadre les collectivités territoriales, et qui, dans les campagnes nigériennes, a quasiment abdiqué de ces diverses fonctions? 
Certes on ne manque pas de "signes extérieurs d'Etat" dans les campagnes. Le sous-préfet fait ses tournées, les écoles tournent tant bien que mal, le tissu encore lâche des dispensaires se resserre peu à peu, les gendarmes arrêtent de temps en temps les fauteurs de troubles. L'Etat existe, puisque ses institutions et ses agents sont visibles. Mais c'est à de nombreux égards devenu une coquille vide, remplissant de plus en plus mal ses missions. Le "système des projets", qui pour une part entend pallier aux insuffisances et dysfonctionnements de l'Etat en milieu rural, n'est pas sans souligner et sans reproduire cet affaiblissement de la puissance publique, puisqu'il crée autant de "micro-para-Etats" éphémères. ostensiblement plus riches et fonctionnels que l'Etat lui-même.

Les projets contre la société civile?

104 L'objectif qui sous-tend de nombreux projets est de se porter directement à la rencontre de la société civile (l'argumentation en oppose les dynamismes à l'impéritie de l'Etat) et de la renforcer. Il n'est pas sûr que le type de société civile ainsi recherchée désespérément existe vraiment, ni que la démarche des projets contribue à la constituer.

Certes les sociétés villageoises ne sont pas amorphes et inorganisées, loin de là. Mais elles sont finalement peu organisées en vue de l'action collective, en particulier à l'échelle communautaire, et le boom associatif qui saisit les classes moyennes africaines urbaines (souvent comme réponse à cette quête d'interlocuteurs collectifs propre à de nombreux bailleurs de fonds) ne se manifeste pas dans les campagnes du Niger.

Il n'existe en fait que deux types de "groupements". Les uns sont "informels", de type plutôt "traditionnel", et sans visée "communautaire". Les autres sont créés de toutes pièces par les projets.

Les groupements informels sont, dans le domaine économique, les tontines et les groupes de travail de jeunes ${ }^{42}$.

108 Contrairement à ce qu'on croit, une tontine (adashe) est plus le produit d'une femme "entrepreneuse" (d'une dynamique individuelle) que l'expression d'une "dynamique collective". La "mère de la tontine". c'est la femme qui prend l'initiative de l'organiser, et de mobiliser un réseau de parentes. voisines, relations. Elle garde l'argent, relance les cotisants, et en retire un petit bénéfice régulier (chacune lui donne "quelque chose" lorsqu'elle touche son tour). La mise en place sur plusieurs années de tontines "améliorées" (asusu) par Care semble un succès. Ces tontines utilisent le capital commun pour faire des crédits (contrairement aux tontines "traditionnelles" ${ }^{43}$ ), et ne le répartissent, avec les bénéfices, qu'au bout de plusieurs mois. Une évaluation de l'impact réel de cette innovation reste cependant à faire.

109 Les groupes de travail de jeunes se constituent entre membres d'une même classe d'âge, sur base d'affinités, et donc rarement à l'échelle du village ${ }^{44}$ La culture d'un champ collectif, ou la "location" du groupe par un cultivateur, procure des ressources communes en général dépensées pour des fêtes. Nous ne connaissons pas d'exemple d"'amélioration" ou d'utilisation de ces structures informelles par des projets ou des services techniques.

110 Sinon, les activités économiques ne s'exercent que sur base individuelle et familiale, ou sous forme de réseaux.

111 On voit bien sûr de plus en plus de "groupements" ou comités" se mettre en place à l'initiative de projets (qui prennent ainsi le relais de l'Etat, qui, après l'indépendance, 
avait implanté partout des "coopératives"). Mais il s'agit de structures qui sont le produit d'initiatives extérieures, et non pas de "dynamiques endogènes". L'objectif des projets est que ces structures soient "appropriées" et débouchent sur des dynamiques locales. Est-ce un souhait réaliste?

Il est difficile de répondre à une telle question. Cela en tout cas ne va pas de soi. contrairement à ce que semblent croire certains projets. Les "groupements" et "comités" ne sont pas automatiquement l'embryon d'une société civile organisée en vue du développement, et ne sont pas nécessairement un pas en avant vers la création d'une dynamique communautaire et municipale. Ils n'ont aucune légitimité en tant qu'expression d'une gestion collective des affaires villageoises, mais représentent simultanément une contrainte imposée par les intervenants et une opportunité pour ceux qui se sont mis en bons termes avec ces intervenants. Ils sont souvent perçus dans les villages comme des groupes d'intérêt particuliers, constitués par ceux qui ont su se positionner comme les interlocuteurs locaux des projets, pour chercher à s'approprier les ressources de ces projets. En ce cas, la constitution de ces groupements et comités aiguise les rivalités et les tensions. On pourrait même soutenir qu'ils bloquent de ce fait l'émergence d'un sens du bien public et de l'intérêt collectif, qu'ils freinent un éventuel processus de "municipalisation". Les projets en sont d'autant moins avertis que la pratique du double langage est assez générale : langage du consensus, du bien du village, de la dynamique collective, en direction des projets (les paysans ayant depuis longtemps appris ce qui plait aux intervenants extérieurs), langage de la concurrence, du soupçon, de la méfiance, dans les interactions quotidiennes au village. Il existe cependant diverses formes d'équilibre entre ces deux tendances opposées, et il est parfois des comités qui reflètent aussi un certain consensus villageois: mais il s'agira plus d'exception que de règle.

113 Par ailleurs, on ne peut non plus éliminer complètement l'hypothèse selon laquelle, malgré tout, ces comités seraient un lieu de formation à la gestion pour de futures "élites municipales paysannes".

Les projets contre les initiatives?

114 Les initiatives "hors projets" sont nombreuses, et elles sont un facteur important du changement social dans les villages. Sans même parler du commerce et de l'accroissement spectaculaire de la consommation marchande dans les villages, on constate que de nombreuses innovations ont été introduites dans les villages par des circuits non institutionnels: moulins privés, puits privés, charrettes, médicaments modernes de colportage, vélos, filets et hameçons, maisons en dur ou semi-dur, etc... Parmi les pratiques agro-pastorales et artisanales un certain nombre, même lorsqu'elles apparaissent à l'extérieur comme "traditionnelles" parce qu'elles ne font pas appel à des techniques nettement "modernes", sont en fait nouvelles, soit parce qu'elles ne se pratiquaient pas auparavant localement, soit parce qu'elles sont passées de l'auto-consommation à la vente et ont changé d'échelle: souchet, niébé, nattes et cordes, pêche, mirici (doum), etc.

115 La concentration des projets sur quelques thèmes standards récurrents contraste ainsi avec la variété des micro-innovations locales, souvent très diversifiées en fonction de l'environnement naturel et commercial local. Leur insistance sur la mise en place de structures collectives, difficiles à faire fonctionner et complexes à gérer, contraste avec la souplesse des initiatives paysannes individuelles. Leur rhétorique de l'"appui" se 
traduit fort peu dans les faits: les projets tendent à n'appuyer que ce qu'ils ont eux-même préalablement pensé et créé.

116 Certes les trois questions posées (les projets contre l'Etat, contre la société civile et contre l'initiative?) sont quelque peu provocantes, et donc excessives. Même si les enquêtes menées dans les quatre sites et nos autres travaux ailleurs au Niger fournissent divers exemples pour les étayer, ces question doivent être prises comme des questions, et non des réponses. Il serait injuste de prendre trop systématiquement le contre-pied des objectifs et des idéologies des projets pour affirmer que ceux-ci ont des conséquences involontaires systématiquement négatives. Il s'agit, avec de telles questions, d'attirer l'attention sur les effets pervers au moins potentiels du système des projets, compte-tenu du contexte très particulier des arènes politiques locales spécifiques aux villages nigériens (faible structuration, concentration sur la chefferie, pas ou peu de modes d'organisation et de gestion d'un bien public). Nous pensons en effet que le développement d'un processus de "municipalisation villageoise" est un enjeu central pour l'avenir, que les campagnes nigériennes en sont encore loin, et que les conditions de son émergence sont particulièrement complexes.

\section{BIBLIOGRAPHIE}

Bailey, F. 1969. Stratagems and spoil. A social anthropology of politics. London : Basil Blackwell (trad. fr. 1971 : Les règles du jeu politique).

Berche, T. 1998. Anthropologie et santé publique : un projet en pays dogon. Paris : APAD-Karthala.

Bierschenk, T. \& J.P. Olivier de Sardan 1997. "ECRIS : Rapid Collective Inquiry for the Identification of Conflicts and Strategie Groups". Human Organization 56 (2) : 238-244.

Doka, M. et al. 1996. Identification des dynamiques locales. région de Maradi. Niamey : BUCO.

Dunbar, R. 1970. Damagaram (Zinder. Niger). 1812-1906 : The History of a Central Sudanic Kingdom. Los Angeles : University of California (doctoral dissertation).

Gado, B. 1980. "Le Zarmatarey : contribution à l'histoire des populations d'entre Niger et Dallol Mawri". Etudes Nigériennes 45 (Niamey).

Hamani, D. 1989. "Au carrefour du Soudan et de la Berbérie : le sultanat touareg de l'Ayar". Etudes Nigériennes (Niamey).

Kaberry, P. \& D. Forde (eds) 1967. West African Kingdoms in the Nineteenth Century. Oxford : Oxford University Press.

Lund, C. 1993. En attendant le code rural : réflexions sur une réforme de la tenure foncière au Niger. London : International Institute for Environment and development (dossier $\left.n^{\circ} 44\right)$.

Lund, C. 1995. "Competition over jurisdictions and political manoeuvring in Niger". Bulletin de l'APAD 9.

Médard, J.-F. (dir.) 1991. Etats d'Afrique Noire : formation. mécanismes et crise. Paris : Karthala. 
Miles, W. 1987. Partitioned Royalty : The Evolution of Hausa Chiefs in Nigeria and Niger". Journal of Modern African Studies 25 (2) : 233-258.

Olivier de Sardan, J.-P. 1984. Les sociétés songhay-zarma. Chefs. esclaves. guerriers. paysans... Paris : Karthala.

Olivier de Sardan, J.-P. 1995. Anthropologie et développement. Essai en socio-anthropologie du changement social. Paris : APAD-Karthala.

Olivier de Sardan, J.-P. 1996. "L'économie morale de la corruption". Politique Africaine 63 : 97-116.

Raynaut, C. 1977. "Circulation monétaire et évolution des structures socio-économiques chez les Haoussas du Niger". Africa 47 (2) : 160-171.

Raynaut, C. 1989. "L'opération de développement et les logiques du changement : la nécessité d'une approche holistique. L'exemple d'un cas nigérien". Genève Afrique 27 (2) : 8-38.

Robinson, P. 1981. African Traditional Rulers and the Modern State: The Linkage Role of Chiefs in the Republic of Niger. Ann Arbor (Michigan) : University Microfilms International.

Rothiot, J.-P. 1988. L'ascension d'un chef africain au début de la colonisation. Aouta, le conquérant (Dosso, Niger). Paris : L'Harmattan.

Salifou, A. 1971. "Le Damagaram ou sultanat de Zinder au XIXe siècle". Etudes nigériennes 27 Niamey.

Smith, M.G. 1967. "A Hausa Kingdom : Maradi under Dan Baskore, 1854-1875". In : Kaberry \& Forde (eds.).

Tidjani Alou, M. 1998. Dynamique de l'Etat post-colonial au Niger. Ms.

Weber, M. 1971. Economie et société. Paris : Plon.

\section{NOTES}

1.L'étude, commandée par le Bureau de Coordination de la Coopération suisse au Niger (BUCO), a porté sur "Les pouvoirs locaux et dynamiques innovatives dans les régions de Maradi et Gaya". Les deux derniers sites sont, parmi d'autres, l'objet d'interventions du PAIGLR, projet financé par la coopération suisse ; à Shadakori la coopération suisse a implanté un couple d'animateurs.

2.La procédure ECRIS combine enquête collective rapide intensive et enquêtes individuelles plus approfondies. Pour une présentation de cette procédure, cf. Bierschenk \& Olivier de Sardan 1997.

3.Les monographies de chaque site ont été faites par quatre jeunes chercheurs nigériens, Moumouni Adamou, Souley Aboubakar, Aboubakar Tidjani AJou, et Chaibou Adamou et sont à la base du présent texte. Ont participé en outre à toute la phase collective de l'enquête Marthe Doka, sociologue à l'ISH et consultante du BUCO, Ibrahim Saadou, doctorant à l'EHESS, et Sanda Maman Sani, chargé de mission à l'IUED (Niger). Nous avions également mené une autre enquête ECRIS un an auparavant dans trois villages de l'Ouest nigérien (Goutoumbou, Guilléni, Kolo Bossey), pour un projet de recherche géré par l'IUED et également financé par le BUCO, avec Moumouni Adamou, Souley Aboubakar et Hamadou Seyni (pour les monographies par site), Mahaman Tidjani Alou et Claudio Tognola (pour la phase collective). Je voudrais remercier tout particulièrement Adamou Moumouni, Souley Aboubacar, Marthe Doka, Mahaman 
Tidjani Alou et Giorgio Bianchi pour leurs remarques et leurs apports à des titres divers, dont cet article garde de nombreuses traces.

4.Le Niger est un des rares pays d'Afrique à n'avoir pas encore mis en œuvre une politique de décentralisation, même si un projet est actuellement prêt, qui combine décentralisation et redécoupage des cantons et circonsriptions.

5.A différentes époques. sous la colonisation, et après l'indépendance, les chefs ont été choisis directement par le pouvoir au sein des prétendants, sans élection. Le texte officiel en vigueur concernant la chefferie (établi pendant la transition démocratique) est l'ordonnance $n^{\circ}$ 93-28 du 30 mars 1993 portant statut de la chefferie traditionnelle au Niger.

6.Nous n'évoquerons pas ici la réalité des chefferies pré-coloniales, qui renvoie à un tout autre contexte (guerres, esclavage, sujétions politiques d'un autre type, etc.) et à des modes de légitimation en fait très différents (cf. pour les sociétés hausa pré-coloniales Dunbar 1970, Hamani 1989, Salifou 1971, Smith 1967, et pour les sociétés songhay-zarma pré-coloniales Gado 1980, Olivier de Sardan 1984).

7.En ce qui concerne les chefferies administratives sous la colonisation et leurs manipulations des légitimités "traditionnelles", cf. Miles 1987, Rothiot 1988, Olivier de Sardan 1984). Notre analyse ici ne portera que sur les chefferies administratives post-coloniales (cf. également le numéro 38, 1990, de Politique Africaine sur le Niger). 8.Cf. les travaux en cours de Mahaman Tidjani Alou. Celui-ci parle par ailleurs d'une "capture" de l'Etat par un groupe social (Tidjani Alou 1998).

9.L'histoire politique du Niger sous la colonisation montre que le succès du RDA s'est fait en grande partie contre la chefferie, qui avait plutôt partie liée avec l'administration coloniale. Il y aurait donc une déjà ancienne tradition de double légitimité : d'un côté celle, nationale, des partis (validée par les élections), de l'autre celle, locale, des chefs (validée par les investitures).

10.C'est plutôt vers l'Est que, à l'image des grandes chefferies pré-coloniales hausa, se multiplient les signes ostentatoires du pouvoir et le nombre des dignitaires de la cour.

11.Cf Rothiot 1988 : et Olivier de Sardan 1984.

12.Cf. Weber 1971.

13.Cf. Médard 1991 ; pour la différence entre nonne officielle et norme pratique, cf. Bailey 1969.

14.Sur l'économie morale de la corruption, cf. Olivier de Sardan 1996 (texte hélas largement tronqué par Politique Africaine...).

15.Dans les villes ou les chefs-lieux d'arrondissement, la présence d'une certaine masse de fonctionnaires, pour qui l'appartenance partidaire investit plus l'ensemble de la sociabilité, peut entrainer une certaine insertion des partis dans le débat local.

16.Parfois, l'imamat se transmet héréditairement, au sein d'une même famille.

17. Ceci ne veut pas dire qu'il n'y a pas de conséquences sociales indirectes : les "isalistes" s'attaquent aux cérémonies traditionnelles, aux dépenses ostentatoires, et voilent les femmes... Au-delà même de cette secte particulière, la pression d'un islam intransigeant prêchant un certain ordre moral se fait sentir un peu partout (cf. le développement dans certaines familles aisées de la claustration des femmes), mais elle ne débouche sur aucun projet politique à l'échelle villageoise, pour le moment en tout cas.

18.Quand les marabouts ou magiciens divers "renforcent" le pouvoir par leurs charmes ou talismans, ils agissent en tant que fournisseurs ou conseillers personnels du chef (ou 
de ses rivaux) ; ce rôle est inscrit dans l'institution politique de la chefferie et des luttes dont elle est l'enjeu, et n'est en rien un centre de pouvoir autonome.

19.Nous verrons plus loin qu'il existe soit des formes "informelles" ou "spontanées" d'organisation (groupes de travail collectif, tontines), soit des formes "modernes" (comités, associations) induites par les projets ; mais les unes n'interviennent pas dans la vie publique et les autres restent très largement soutenues à bout de bras par les projets...

20.Il contraste avec les mythes communautaires facilement plaqués sur les villages africains.

21.Il y a certainement une inégalité selon les régions et les villages, entre autre due aux moyens d'accès. Il est clair que les villages du Nord du Zarmaganda, pourtant parmi les plus démunis du pays, voient plus rarement que les autres passer des $4 \times 4$ aux portières ornées de sigles divers...

22.La pérennisation pourrait être envisagée autrement qu'à travers des structures, par exemple à travers

des hommes ou des pratiques...

23.L'appel épisodique à des chercheurs (qui reste rare), ou le recours (devenu quasi systématique) à des méthodes de diagnostic dit participatif (MARP ou autres) ne règle pas nécessairement le problème. Il nous faut toutefois préciser que la coopération suisse au Niger, qui a par ailleurs commandé cette étude, se distingue par un recours régulier à des compétences sociologiques fines.

24.Fréquemment les projets laissent aux groupements ou coopératives la disposition de la somme correspondant aux intérêts, et ne récupèrent que le capital; mais cette somme (un peu comme les ristournes de coton) est souvent considérée comme n'appartenant à personne et donc souvent "appropriée" par le comité de gestion, le président, le trésorier...

25.La progression spectaculaire des écoles coraniques, parallèlement aux difficultés croissantes du système d'enseignement officiel et au peu de débouchés qu'il procure à la sortie du primaire, aboutit à ce qu'il y a, dans deux sur quatre des sites enquêtés, nettement plus d'élèves scolarisés en arabe qu'en français.

26.Mais le site d'implantation d'un puits en ciment (ou d'une pompe), choisi parfois plus ou moins arbitrairement par les opérateurs, parfois sur conseil du chef, est une fréquente source de conflits entre quartiers et de soupçons de favoritisme ou de corruption.

27.En particulier dans la zone de Maradi, rappelons que le PDRM aurait eu de 1977 à 1988 un budget annuel de près de 1,8 milliards CFA avant dévaluation, soit 3,6 milliards CFA actuels...

28.Cette technique était déjà anciennement pratiquée, mais seulement sur les champs proches des habitations.

29.Les contrats de fumure avec les pasteurs sont toujours pratiqués, mais il s'agit là d'une pratique très ancienne.

30.Cf. Hambally Yacouba (thèse en cours).

31.Sur les sites d'enquête nous n'avons pas constaté de tentatives de confection de "demi-lunes" contre l'érosion.

32.Certes, face à des personnes venues de l'extérieur et dont on pense qu'elles sont liées à des projets ou peuvent en amener, les villageois pratiqueront généralement une stratégie de mise en scène de l'harmonie et du consensus villageois. Mais c'est l'avantage de la procédure ECRIS que d'aller au-delà de cette façade... 
33.Sur les modes d'appropriation-détournement des projets par telle ou telle fraction des populations, cf. Olivier de Sardan 1995.

34.Sur la question des per-diem et suppléments de salaire ("topping up") dans un projet - en l'occurrence au Mali - , cf. Berche 1998.

35.Et même plutôt contre eux, puisqu'elle épuise rapidement les sols.

36.Cf. Raynaut 1989.

37.On a parlé à juste titre d'une "interpénétration des systèmes de production" entre agriculture et élevage : cf. Doka et al. 1996.

38.Cf. un travail sur les conflits fonciers, qui s'appuie sur des exemples pris dans la région de Zinder mais dont les analyses valent souvent pour tout le pays (Lund 1993, 1995).

39.Notons que dans ce dernier cas la protection des rôneraies n'est pas assortie d'un véritable plan de gestion et d'exploitation de la ressource.

40.Cf. Raynaut 1977.

41.Les groupements ou coopératives, au contraire, achètent parfois des vivres pour spéculer...

42.Il y a bien sûr dans les villages d'autres types de "groupements informels", extérieurs au domaine économique : les confréries islamiques, les confréries de possession, les groupes de baptêmes...

43.Dans beaucoup de villages les tontines "traditionnelles" ne sont pas pré-coloniales et datent en fait de quelques années ou dizaines d'années...

44.La samaria dans les années 70-80 était une exception, mais nous avons vu que sous cette forme moderne elle était le produit d'une mobilisation politique venue d'en haut. Le rapport avec la samaria traditionnelle d'antan est une autre histoire.

\section{AUTEUR}

\section{JEAN-PIERRE OLIVIER DE SARDAN}

EHESS-CNRS, 2 rue de la Vieille Charité, F-13002 Marseille (France). Tel. +33 (4) 91 140753. Fax. +33 (4) 91913401 - jpos@ehess.cnrs-mrs.fr 